\title{
Associação de herbicidas para o controle de plantas daninhas em feijão do tipo preto
}

\section{Herbicide association applied to control weeds in bean of black type}

\section{Leandro Galon ${ }^{1 *}$, Fabio Luís Winter ${ }^{2}$, César Tiago Forte ${ }^{3}$, Luciane Renata Agazzi²,} Felipe José Menin Basso², Cinthia Maethê Holz², Gismael Francisco Perin²

Resumo - O feijão do tipo preto é um dos principais grãos cultivados no Brasil, com importância econômica, social e cultural. As perdas de produtividade de grãos, por competição com as plantas daninhas, em especial Digitaria ciliaris (milhã/capim-colchão) e Urochloa plantaginea (capim-marmelada/papuã) podem chegar a níveis de até $80 \%$ caso nem um método de controle for adotado. Buscando o controle dessas plantas daninhas, objetivou-se com o trabalho avaliar a eficácia e a seletividade de herbicidas aplicados em pré e pós-emergência do feijão do tipo preto. O delineamento experimental utilizado foi de blocos casualizados, com quatro repetições. Os herbicidas utilizados foram: fomesafen (250 $\mathrm{g} \mathrm{ha}^{-1}$ de i.a), s-metolachlor (1200 $\mathrm{g} \mathrm{ha}^{-1}$ de i.a), oxifluorfen (480 $\mathrm{g} \mathrm{ha}^{-1}$ de i.a), clomazone (1000 $\mathrm{g} \mathrm{ha}^{-1}$ de i.a), pendimentalin (1000 $\mathrm{g} \mathrm{ha}^{-1}$ de i.a), flumioxazin ( $25 \mathrm{~g} \mathrm{ha}^{-1}$ de i.a), fluazifop-p-buthyl (250 $\mathrm{g} \mathrm{ha}^{-1}$ de i.a), imazamox (42 $\mathrm{g} \mathrm{ha}^{-1}$ de i.a), bentazon (720 $\mathrm{g} \mathrm{ha}^{-1} \mathrm{de}$ i.a), bentazon + imazamox $\left(600+28 \mathrm{~g} \mathrm{ha}^{-1} \mathrm{de}\right.$ i.a $)$, fluazifop-p-buthyl + fomesafen $\left(250+250 \mathrm{~g} \mathrm{ha}^{-1} \mathrm{de}\right.$ i.a), seguidos de mais duas testemunhas, uma capinada e outra infestada. O oxifluorfen apresentou os maiores níveis de fitotoxicidade ao feijoeiro cultivar BRS Campeiro, o que resultou no efeito negativo sobre os componentes do rendimento de grãos, juntamente com a testemunha infestada, o s-metolachlor, o pendimentalin, o flumioxazin, o fomesafen e o bentazon. A mistura comercial composta de bentazon + imazamox apresentou baixa fitotoxicidade, os melhores resultados em relação aos componentes do rendimento de grãos, aliado a um bom controle da milhã e do papuã. Palavras-chave: Digitaria ciliaris, Phaseolus vulgaris, Urochloa plantaginea.

Abstract - Black bean is one of the main grains grown in Brazil, with economic, social and cultural importance. Grain productivity losses due to competition with weeds, in particular Digitaria ciliaris (summergrasster) and Urochloa plantaginea (signalgrass) can reach levels of up to $80 \%$ if neither Control is adopted. The objective of this study was to evaluate the efficacy and selectivity of herbicides applied in pre and post emergence of black beans. The experimental design was a randomized block design, with four replications. The herbicides used were fomesafen (250 $\left.\mathrm{g} \mathrm{ha}^{-1} \mathrm{a} . \mathrm{i}\right)$, s-metolachlor (1200 $\left.\mathrm{g} \mathrm{ha}^{-1}\right)$, oxyfluorfen (480 $\mathrm{g} \mathrm{ha}^{-1}$ of a.i), clomazone (1000 $\mathrm{g} \mathrm{ha}^{-1}$ of a.i), pendimentalin (250 $\mathrm{g} \mathrm{ha}^{-1}$ of a.i), imazamox (42 $\mathrm{g} \mathrm{ha}^{-1}$ of a.i), bentazon (720 $\left.\mathrm{g} \mathrm{ha}^{-1} \mathrm{of} \mathrm{a.i}\right)$,

Recebido: Agosto 11, 2017. Aceito: Dezembro 05, 2017.

${ }^{1}$ Programa de Pós-graduação em Ciência e Tecnologia Ambiental, Curso de Agromomia, Universidade Federal da Fronteira Sul - UFFS, km 72, ERS 135, n 200, CP 764, CEP 99700-970, Erechim, RS, Brasil.

E-mail: leandro.galon@uffs.edu.br

${ }^{2}$ Curso de Agronomia, Universidade Federal da Fronteira Sul - UFFS, Erechim, RS, Brasil. E-mail: fabioaratiba@gmail.com; luci_agazzi@hotmail.com; felipebasso1@hotmail.com; cinthiaholz@gmail.com; gismaelperin@gmail.com

${ }^{3}$ Programa de Pós-graduação em Ciência e Tecnologia Ambiental, Universidade Federal da Fronteira Sul - UFFS, Erechim, RS, Brasil. E-mail: cesartiagoforte@hotmail.com 
bentazon + imazamox $\left(600+28 \mathrm{~g} \mathrm{ha}^{-1}\right.$ of a.i), flumioxazin $\left(25 \mathrm{~g} \mathrm{ha}^{-1}\right.$ of a.i $)$, fluazifop-p-buthyl + fomesafen $\left(250+250 \mathrm{~g} \mathrm{ha}^{-1}\right.$ of a.i), followed by two additional controls, one weed and one infested. O oxyfluorfen presented the highest levels of phytotoxicity to BRS Campeiro bean, which resulted in a negative effect on components of grain yield, together with the infested control, s-metolachlor, pendimentalin, flumioxazin, fomesafen and bentazon. The commercial mixture of bentazon + imazamox presented low phytotoxicity, the best results of the components of grain yield, together with a good control of the summergrass and the signalgrass.

Keywords: Digitaria ciliaris, Phaseolus vulgaris, Urochloa plantaginea.

\section{Introdução}

A produção de feijão se destaca no cenário brasileiro por apresentar a terceira maior área cultivada na safra 2016/17 com 2993,4 mil ha com estimativa de produção de 3276,7 mil toneladas de grãos, ficando atrás apenas do milho e da soja. A produtividade média brasileira de feijão é de $1.076 \mathrm{~kg} \mathrm{ha}^{-1}$ (CONAB, 2017), sendo essa muito inferior ao observado em áreas experimentais ou em lavouras que adotam altos níveis tecnológicos.

Dentre os fatores que tem acarretado baixas produtividades do feijoeiro destaca-se a interferência das plantas daninhas, por competirem pelos recursos água, luz e nutrientes. Quando não controladas as plantas daninhas ocasionam perdas da produtividade de grãos de até $80 \%$ ou mesmo podem depreciar a qualidade dos grãos colhidos (Vidal et al., 2010; Kalsing e Vidal, 2012), além de serem hospedeiras de insetos e doenças.

As espécies de plantas daninhas, Digitaria ciliaris (milhã ou capim-colchão) e Urochloa plantaginea (capim-marmelada ou papuã), são encontradas em todo o território nacional, em especial no Sul do Brasil, causando severos prejuízos às culturas agrícolas (Fontana et al., 2016; Kalsing e Vidal, 2012). A utilização de herbicidas para o controle de plantas daninhas apresenta maior relevância dentre os demais métodos de manejo, devido à eficácia, rapidez e menor custo quando comparado a outras formas de controle.

$\mathrm{Na}$ atualidade tem-se se observado a ocorrência de plantas daninhas infestantes das lavouras que adquiriram resistência a muitos herbicidas. Desse modo a rotação ou associação de herbicidas de diferentes mecanismos de ação para o controle das plantas daninhas torna-se uma ferramenta fundamental para reduzir a ocorrência da resistência (Brunharo et al., 2014). Destaca-se ainda que se torna importante o uso de herbicidas em pré-emergência das culturas, já que alguns trabalhos vêm mostrando a necessidade do controle das plantas daninhas antes mesmo de emergirem o que tem gerado bons resultados de produtividade de grãos (Sikkema et al., 2008; Silva et al., 2014).

As pesquisas tem demonstrado que vários são os herbicidas que tem apresentado eficácia no controle das plantas daninhas infestantes do feijoeiro, tais como o s-metolachlor (Sikkema et al., 2008; Lamego et al., 2011); bentazon + imazamox e o fomesafen (Soltani et al., 2005; Silva et al., 2013). Enquanto que, outros herbicidas como o diclosulam (Mancuso et al., 2016) e o alachlor (Lamego et al., 2011) demonstraram injúrias indesejáveis na cultura do feijoeiro o que pode comprometer a produtividade de grãos.

$\mathrm{O}$ estudo da associação de herbicidas com diferentes mecanismos de ação se torna importante no cenário produtivo, por identificar quais são capazes de apresentar controle significativo de plantas daninhas sem prejudicar a produtividade das culturas. Além disso, o uso de herbicidas com ação em pré e pós-emergência com diferentes mecanismos de ação, apresentam-se como opção de manejo para aumentar os níveis de controle (Bressanin et al., 2015), contribuindo para reduzir a pressão de seleção e diminuir novos casos de plantas daninhas resistentes. 
A hipótese da presente pesquisa é de que a associação de herbicidas de diferentes mecanismos de ação ocasiona melhor controle de plantas daninhas e não interfere nos componentes de rendimento do feijão do tipo preto.

Diante disso, objetivou-se com o trabalho avaliar a eficácia e a seletividade de herbicidas aplicados em pré e pós-emergência do feijão do tipo preto, cultivar BRS Campeiro.

\section{Material e métodos}

O experimento foi conduzido a campo na área experimental da Universidade Federal da Fronteira Sul (UFFS), Campus Erechim, de novembro de 2015 a fevereiro de 2016. O solo da área experimental é classificado como Latossolo Vermelho Aluminoférrico húmico, Unidade de mapeamento Erechim (EMBRAPA, 2013). A correção do $\mathrm{pH}$ e a adubação do solo foram realizadas de acordo com a análise físico-química e seguindo-se as recomendações técnicas para a cultura do feijão (Rolas, 2004).

A semeadura da cultivar de feijão do tipo preto, BRS Campeiro, foi efetuada em sistema de plantio direto na palha, sendo que 30 dias antes dessa operação efetuou-se a dessecação da vegetação usando-se os herbicidas glyphosate $+2,4-\mathrm{D}$ na dose de $3,0+1,5 \mathrm{~L} \mathrm{ha}^{-1}$, respectivamente.

O experimento foi instalado em delineamento de bloco casualizados, com quatro repetições, sendo os tratamentos, doses e momento de aplicação dos herbicidas descritos na Tabela 1. A pluviosidade média que ocorreu desde a implantação até a colheita da cultura, bem como as temperaturas máximas e mínimas estão representadas na Figura 1.

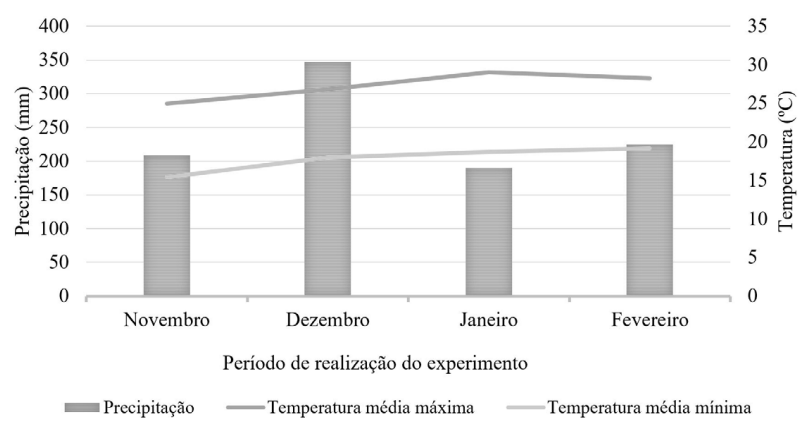

Figura 1. Precipitação pluviométrica mensal (mm), temperatura média máxima e mínima no período de realização do experimento.

Tabela 1. Tratamentos utilizados no experimento, respectivas doses e época de aplicação para controle de plantas daninhas (papuã e milhã) infestantes da cultivar de feijão do tipo preto, BRS Campeiro.

\begin{tabular}{lcccc}
\hline \multicolumn{1}{c}{ Tratamentos } & $\begin{array}{c}\text { Doses }\left(\mathbf{g ~ h a}^{-1}\right) \mathbf{d e} \\
\text { ingrediente ativo (i.a) }\end{array}$ & Nome comercial & Dose & $\begin{array}{c}\text { Época de } \\
\text { aplicação }\end{array}$ \\
\hline Testemunha infestada & $\ldots$ & $\ldots$ & $\ldots$ & $\ldots$ \\
Testemunha capinada & $\ldots$ & $\ldots$ & $\ldots$ & $\ldots$ \\
S-metolachlor + fomesafen & $1200+250$ & Dual Gold+Flex & $1,25+1,00$ & Pré + Pós \\
Oxifluorfen & 480 & Goal BR & 2,00 & Pré \\
S-metolachlor & 1200 & Dual Gold & 1,25 & Pré \\
Clomazone & 720 & Gamit $360 \mathrm{CS}$ & 2,00 & Pré \\
Pendimenthalin & 1000 & Herbadox & 2,00 & Pré \\
Flumioxazin & 25 & Flumyzin 500 & 0,05 & Pré \\
Fluazifop-p-butil & 250 & Fusilade 250 EW & 1,00 & Pós \\
Fomesafen & 250 & Flex & 1,00 & Pós \\
S-metolachlor +imazamox & $1200+42$ & Dual Gold+Sweeper & $1,25+0,06$ & Pré + Pós \\
Bentazon & 720 & Basagran 600 & 1,20 & Pós \\
Imazamox & 42 & Sweeper & 0,06 & Pós \\
Bentazon+imazamox & $600+28$ & Amplo & 1,00 & Pós \\
Fluazifop-p-butil+fomesafen & $250+250$ & Fusiflex & 2,00 & Pós \\
Flumioxazin+fluazifop-p-butil & $25+250$ & Flumyzin 500+Fusilade $250 \mathrm{EW}$ & $0,05+1,00$ & Pré + Pós \\
\hline
\end{tabular}


Os herbicidas utilizados foram: fomesafen (Flex, $250 \mathrm{~g} \mathrm{~L}^{-1}$, SL, Syngenta) s-metolachlor (Dual Gold, $960 \mathrm{~g} \mathrm{~L}^{-1} \mathrm{EC}$, Syngenta), oxifluorfen (Goal BR, $240 \mathrm{~g} \mathrm{~L}^{-1}$, EC, Dow Agrosciences), clomazone (Gamit $360 \mathrm{CS}, 360 \mathrm{~g} \mathrm{~L}^{-1}$, FMC), pendimenthalin (Herbadox, $500 \mathrm{~g} \mathrm{~L}^{-1}, \mathrm{EC}$, Basf), flumioxazin (Flumyzin 500, $500 \mathrm{~g} \mathrm{~kg}^{-1}$, WG, Sumitomo), fluazifop-p-buthyl (Fusilade, $250 \mathrm{~g} \mathrm{~L}^{-1}, \mathrm{EW}$, Syngenta), imazamox (Sweeper, $700 \mathrm{~g} \mathrm{~kg}^{-1}$, WG, Basf), bentazon (Basagran 600, $\left.600 \mathrm{~g} \mathrm{~L}^{-1}, \mathrm{SL}, \mathrm{Basf}\right)$, bentazon + imazamox (Amplo, $\left.600+28 \mathrm{~g} \mathrm{~L}^{-1}, \mathrm{SL}, \mathrm{Basf}\right)$, fluazifop-p-buthyl + fomesafen (Fusiflex, $125+125 \mathrm{~g} \mathrm{~L}^{-1}$, SL, Syngenta). Ressalta-se que alguns dos herbicidas testados no referido experimento não estão registrados para serem aplicados no controle de plantas daninhas infestantes do feijão, no entanto busca-se também com o presente estudo informações em relação a seletividade e a eficácia para se ter novas opções para o controle químico nessa cultura. Cada herbicida recebeu o adjuvante recomendado pelo respectivo fabricante.

A adubação química no sulco de semeadura foi de $260 \mathrm{~kg} \mathrm{ha}^{-1}$ da fórmula 05-20-20 de N-P-K e aplicação de nitrogênio em cobertura foi realizada no estádio de V3 (primeiro trifólio desenvolvido) da cultura, na dose de $67,5 \mathrm{~kg} \mathrm{ha}^{-1}$ de N. Cada unidade experimental foi caracterizada por uma parcela de $15 \mathrm{~m}^{2}$ ( $\left.5 \times 3 \mathrm{~m}\right)$ semeadas com 6 linhas de feijão do tipo preto em espaçamento de $0,5 \mathrm{~m}$ com uma população de 10 plantas $\mathrm{m}^{-1}$.

A aplicação dos herbicidas foi efetuada com auxílio de um pulverizador costal de precisão, pressurizado a $\mathrm{CO}_{2}$, equipado com quatro pontas de pulverização tipo leque DG 110.02, mantendo-se a pressão constante de $210 \mathrm{kPa}$ e velocidade de deslocamento de $3,6 \mathrm{~km} \mathrm{~h}^{-1}$, o que proporcionou a vazão de $150 \mathrm{~L} \mathrm{ha}^{-1}$ de calda de herbicida.

As condições ambientais no momento da aplicação dos herbicidas em pré-emergência eram: céu nublado, temperatura do ar de $21,3^{\circ} \mathrm{C}$, umidade relativa do ar de $78 \%$, solo úmido e ventos de 4 a $8 \mathrm{~km} \mathrm{~h}^{-1}$. Já na aplicação em pós-emergência o céu apresentava-se parcialmente nublado, temperatura do ar de $27,2^{\circ} \mathrm{C}$, umidade relativa do ar de $61 \%$, solo úmido e ventos de 1,4 a 3,4 $\mathrm{km} \mathrm{h}^{-1}$. A cultura do feijão encontra-se com 2 trifólios completamente desenvolvidos (estádio V4), as plantas daninhas; papuã com 2 folhas a 3 perfilhos e a milhã com 2 folhas a 2 perfilhos no momento da aplicação dos herbicidas em pós-emergência. As densidades médias das plantas daninhas na área experimental eram de 240 e de 40 plantas $\mathrm{m}^{-2}$ de papuã e milhã, respectivamente.

A fitotoxicidade ocasionada pelos herbicidas a cultivar de feijão do tipo preto BRS Campeiro foi avaliada aos 7, 14, 21 e 28 dias após a aplicação dos tratamentos (DAT). Já as avaliações de controle do papuã e da milhã foram realizadas aos 7, 14, 21, 28 DAT e na pré-colheita da cultura. Foram atribuídas notas percentuais, em que zero $(0 \%)$ representava ausência de fitotoxicidade ao feijão, ou com ausência de controle do papuã e/ou da milhã e a nota de cem (100\%) à morte completa da cultura ou das plantas daninhas (SBCPD, 1995).

As variáveis avaliadas, em 10 plantas de cada parcela, na pré-colheita do feijão foram: o número de vagens por planta (NVP) e o número de grãos por vagens (NGV). Nessa mesma época determinou-se ainda no centro de cada parcela o número de plantas (NP) por metro linear. A colheita do feijão foi realizada quando os grãos atingiram $16 \%$ de umidade, em área útil de 3,0 $\mathrm{m}^{2}$ por unidade experimental, efetuando-se posteriormente a trilha. Por fim determinou-se ainda a massa de mil grãos - MMG (g), contando-se 8 amostras de 100 grãos cada e pesando-se as mesmas em balança analítica. Para as análises, a umidade dos grãos foi ajustada para $13 \%$ e os dados de produtividade (PROD) extrapolados para $\mathrm{kg} \mathrm{ha}^{-1}$.

Os dados foram submetidos à análise de variância pelo teste $\mathrm{F}$, em sendo significativos as médias foram comparadas pelo teste de Tukey a $\mathrm{p}<0,05$.

\section{Resultados e discussão}

Os resultados demonstram que o herbicida oxifluorfen apresentou as maiores fitotoxicidades a cultivar de feijão do tipo preto, BRS Campeiro, 
em todas as épocas avaliadas (Tabela 2). Alguns herbicidas apresentaram as maiores fitotoxicidades aos 7 dias após a aplicação (DAT), com recuperação dos sintomas de injúrias com o passar do tempo, como é o caso do fluazifop-p-buthyl, fomesafen, bentazon, imazamox, bentazon + imazamox e fluazifop-p-buthyl + fomesafen. Algumas pesquisas tem relatado que a aplicação de imazamox, bentazon, fluazifop-p-butil e fomesafen em feijoeiro tem ocasionado injúrias, porém são detoxificadas e a cultura se recupera em poucos dias após a aplicação (Soltani et al., 2005; Nina, 2011; Fontes et al., 2013; Zargar et al., 2014), o que condiz com os resultados encontrados no presente estudo.

Na avaliação dos 14 DAT, novamente o herbicida oxifluorfen apresentou a maior fitotoxicidade ao feijão, devido, possivelmente ao seu potencial de controle de dicotiledôneas (Rodrigues e Almeida, 2011). Os demais herbicidas não diferiram entre si, sendo que o maior sintoma de fitotoxicidade não ultrapassou os 6\% (Tabela 2). Os herbicidas mais utilizados para o controle de plantas daninhas em feijão (fomesafen e fluazifop-p-butil) por controlarem mono e dicotiledôneas, apresentam considerável fitotoxicidade inicial, porém a cultura foi capaz de se recuperar dos sintomas de injúrias conforme foi se desenvolvendo, fato esse também constatado por Oliveira et al. (2013).

Aos 21 e 28 DAT observou-se que o fomesafen + s-metolachlor, s-metolachlor, clomazone, pendimenthalin, flumioxazin, imazamox + s-metolachlor e flumioxazin + fluazifop-p-butil foram os herbicidas que apresentaram os maiores sintomas de fitotoxicidades ao serem comparados com as testemunhas capinada e infestada, no entanto mesmo assim ocasionaram menores injúrias ao feijão em relação ao oxifluorfen (Tabela 2). Mesmo que o fomesafen + s-metolachlor, s-metolachlor, clomazone, pendimenthalin, flumioxazin, imazamox + s-metolachlor e flumioxazin + fluazifop-p-butil tenham ocasionado as maiores fitotoxidades, os sintomas não ultrapassaram os $10 \%$, índice esse considerado baixo. Ao aplicarem diferentes

Tabela 2. Fitotoxicidade (\%) a cultivar de feijão do tipo preto, BRS Campeiro em função da aplicação de herbicidas em pré e pós-emergência.

\begin{tabular}{|c|c|c|c|c|}
\hline \multirow{2}{*}{ Tratamentos } & \multicolumn{4}{|c|}{ Fitotoxicidade (\%) } \\
\hline & 7 DAT $^{1}$ & 14 DAT & 21 DAT & 28 DAT \\
\hline Testemunha infestada & $0 \mathrm{~h}^{2}$ & $0 \mathrm{c}$ & $0 \mathrm{e}$ & $0 \mathrm{c}$ \\
\hline Testemunha capinada & $0 \mathrm{~h}$ & $0 \mathrm{c}$ & $0 \mathrm{e}$ & $0 \mathrm{c}$ \\
\hline Fomesafen + s-metolachlor ${ }^{3}$ & $3 \mathrm{efgh}$ & $5 \mathrm{~b}$ & $7 \mathrm{c}$ & $4 \mathrm{~b}$ \\
\hline Oxifluorfen $^{3}$ & $56 \mathrm{a}$ & $22 \mathrm{a}$ & $56 \mathrm{a}$ & $34 \mathrm{a}$ \\
\hline S-metolachlor ${ }^{3}$ & $2 \mathrm{fgh}$ & $4 \mathrm{~b}$ & $5 \mathrm{~cd}$ & $5 \mathrm{~b}$ \\
\hline Clomazone $^{3}$ & 3 efgh & $6 \mathrm{~b}$ & $4 \mathrm{~d}$ & $4 \mathrm{~b}$ \\
\hline Pendimenthalin ${ }^{3}$ & $1 \mathrm{gh}$ & $4 \mathrm{~b}$ & $5 \mathrm{~cd}$ & $5 \mathrm{~b}$ \\
\hline Flumioxazin ${ }^{3}$ & $1 \mathrm{gh}$ & $5 \mathrm{~b}$ & $4 \mathrm{~d}$ & $5 \mathrm{~b}$ \\
\hline Fluazifop-p-butil & 6 defg & $4 \mathrm{~b}$ & $0 \mathrm{e}$ & $0 \mathrm{c}$ \\
\hline Fomesafen & $9 \mathrm{~cd}$ & $3 \mathrm{bc}$ & $0 \mathrm{e}$ & $0 \mathrm{c}$ \\
\hline Imazamox + s-metolachlor ${ }^{3}$ & $1 \mathrm{gh}$ & $4 \mathrm{~b}$ & $10 \mathrm{~b}$ & $4 \mathrm{~b}$ \\
\hline Bentazon & $7 \mathrm{cde}$ & $4 \mathrm{~b}$ & $0 \mathrm{e}$ & $0 \mathrm{c}$ \\
\hline Imazamox & $12 \mathrm{c}$ & $4 \mathrm{~b}$ & $0 \mathrm{e}$ & $0 \mathrm{c}$ \\
\hline Bentazon + imazamox & $7 \mathrm{cde}$ & $3 \mathrm{bc}$ & $0 \mathrm{e}$ & $0 \mathrm{c}$ \\
\hline Fluazifop-p-butil + fomesafen & $18 \mathrm{~b}$ & $4 \mathrm{~b}$ & $0 \mathrm{e}$ & $0 \mathrm{c}$ \\
\hline Flumioxazin $^{2}+$ fluazifop-p-butil & 3 efgh & $6 \mathrm{~b}$ & $5 \mathrm{~cd}$ & $5 \mathrm{~b}$ \\
\hline CV $(\%)$ & 24,88 & 26,80 & 13,26 & 18,23 \\
\hline
\end{tabular}

${ }^{1}$ Dias após a aplicação dos tratamentos; ${ }^{2}$ Médias seguidas de mesmas letras minúsculas na coluna não diferem entre si pelo teste de Tukey a $\mathrm{p}<0,05 ;{ }^{3}$ Aplicação do herbicida em pré-emergência da cultura. 
herbicidas em feijão-caupi Fontes et al. (2013) observaram que o bentazon e o fluazifop-p-butil não demonstraram sintomas elevados de fitotoxicidade aos 21 DAT. Nina (2011) ao aplicar oxifluorfen em feijão-caupi relata que esse herbicida ocasionou a morte das plantas não sendo assim recomendado o uso desse produto para o controle de plantas daninhas infestantes do feijão. Em feijão-caupi a utilização de bentazon + imazamox apresentou leve fitotoxicidade aos 3 DAT com recuperação total das anomalias aos 15 DAT e o uso de fomesafen ocasionou injúrias mais severa (Linhares et al., 2014), resultado esse que não foi observado no feijão do tipo preto.

Os resultados encontrados por outros pesquisadores, relatados anteriormente, corroboram com os demonstrados no presente estudo. Ressalta-se que algumas diferenças que se encontrou em relação a seletividade de herbicidas quando foram aplicados em feijão-caupi e quando usados e comparados com feijão do tipo preto esta associado principalmente pelas características genéticas distintas que essas duas espécies de feijão apresentam, o que pode aumentar ou diminuir a seletividade de culturas a agentes xenobióticos, no caso herbicidas. Vargas e Roman (2005) ao avaliarem a seletividade de herbicidas em diferentes espécies também constataram diferenciação em relação a fitotoxicidade de acordo com o produto aplicado e com a cultura. Santos et al. (2014) relataram que a seletividade de herbicidas em plantas depende de fatores associados as características físico-químicas de cada herbicida, das intrínsecas de cada cultivar e também da dose dos produtos utilizados. Bunting et al. (2004) e Armel et al. (2005) relataram, ao estudarem a seletividade de diferentes cultivares de sorgo a herbicidas, que a tolerância diferencial das cultivares pode ser atribuída a diferente base genética dos genótipos que pode resultar em diferenças morfofisiológicas nas plantas, afetando a absorção, a translocação e o metabolismo dos herbicidas, promovendo dessa forma resposta diferencial das cultivares aos produtos avaliados.
Observou-se nas avaliações efetuadas dos 7, 14, 21 e 28 DAT, e na pré-colheita do feijão que o fluazifop-p-butil e o flumioxazin + fluazifopp-butil apresentaram os melhores resultados de controle das plantas daninhas milhã (Tabela 3) e papuã (Tabela 4), ao igualarem-se esses herbicidas com a testemunha capinada. A mistura formulada comercialmente composta de fluazifop-p-butil + fomesafen apresentou excelente controle da milhã (Tabela 3) do início ao final das avaliações e para o papuã aos 7, 14, 21 e 28 DAT (Tabela 4) não diferindo da testemunha capinada. Levando-se em conta que o período crítico de controle das plantas daninhas infestantes do feijão é de 04 a 18 dias após a emergência (Borchartt et al., 2011) pode-se dizer que essa mistura teria condições de ser utilizada para o controle químico, ainda mais por apresentar dois mecanismos de ação (Inibidor de ACCase - fluazifop-p-butil e de PROTOX - fomesafen) o que dificulta o surgimento da resistência na lavoura, favorecendo assim o manejo das plantas daninhas infestantes do feijoeiro.

Dentre os herbicidas aplicados em pré-emergência o clomazone foi o que apresentou o melhor desempenho em comparação com os demais, com índices de controle elevados inicialmente (acima de $89 \%$ ) e reduzindo com o passar do tempo para $83 \%$ de controle aos 28 DAT (Tabelas 3 e 4 ). O uso de herbicidas pré-emergentes em feijão-caupi, como o s-metolachlor demonstrou ótimo controle das plantas daninhas, Trianthema portulacastrum, Amaranthus spinosus, Commelina benghalensis e Digitaria bicornis (Silva et al., 2014). Fato que no presente experimento só ocorreu aos 14 e 21 DAT para o controle de milhã, onde o s-metolachlor demonstrou controle acima de $83 \%$, o que se considera bom, pois para ser considerado eficiente um herbicida precisa apresentar controle de determinada planta daninha, superior a $80 \%$ (Oliveira et al., 2009). Essa diferenciação da eficácia de um determinado herbicida, em especial os aplicados em pré-emergência, esta ligado a fatores ambientais - índices de precipitação, temperatura, dentre outros, características do 
Tabela 3. Controle (\%) de milhã infestante da cultivar de feijão do tipo preto BRS Campeiro em função da aplicação de herbicidas em pré e pós-emergência.

\begin{tabular}{|c|c|c|c|c|c|}
\hline \multirow{2}{*}{ Tratamentos } & \multicolumn{4}{|c|}{ Controle de milhã (\%) } & \multirow{2}{*}{$\begin{array}{c}\text { Pré } \\
\text { colheita }\end{array}$} \\
\hline & 07 DAT ${ }^{1}$ & 14 DAT & 21 DAT & 28 DAT & \\
\hline Testemunha infestada & $0 \mathrm{~g}^{2}$ & $0 \mathrm{c}$ & $0 \mathrm{f}$ & $0 \mathrm{f}$ & $0 \mathrm{~d}$ \\
\hline Testemunha capinada & $100 \mathrm{a}$ & $100 \mathrm{a}$ & $100 \mathrm{a}$ & $100 \mathrm{a}$ & $100 \mathrm{a}$ \\
\hline Fomesafen + s-metolachlor ${ }^{3}$ & $80 \mathrm{bcd}$ & $90 \mathrm{ab}$ & $85 \mathrm{abcd}$ & $65 \mathrm{de}$ & $0 \mathrm{~d}$ \\
\hline Oxifluorfen ${ }^{3}$ & $0 \mathrm{~g}$ & $0 \mathrm{c}$ & $0 \mathrm{f}$ & $0 \mathrm{f}$ & $0 \mathrm{~d}$ \\
\hline S-metolachlor ${ }^{3}$ & $43 \mathrm{f}$ & $89 \mathrm{ab}$ & $83 \mathrm{~cd}$ & $53 \mathrm{e}$ & $0 \mathrm{~d}$ \\
\hline Clomazone $^{3}$ & $89 \mathrm{abc}$ & $89 \mathrm{ab}$ & 85 abcd & $83 \mathrm{bc}$ & $0 \mathrm{~d}$ \\
\hline Pendimenthalin ${ }^{3}$ & $0 \mathrm{~g}$ & $0 \mathrm{c}$ & $0 \mathrm{f}$ & $0 \mathrm{f}$ & $0 \mathrm{~d}$ \\
\hline Flumioxazin ${ }^{3}$ & $30 \mathrm{f}$ & $0 \mathrm{c}$ & $0 \mathrm{f}$ & $0 \mathrm{f}$ & $0 \mathrm{~d}$ \\
\hline Fluazifop-p-butil & $89 \mathrm{abc}$ & $99 \mathrm{a}$ & $99 \mathrm{ab}$ & $95 \mathrm{ab}$ & $98 \mathrm{a}$ \\
\hline Fomesafen & $59 \mathrm{e}$ & $29 \mathrm{c}$ & $34 \mathrm{e}$ & $0 \mathrm{f}$ & $0 \mathrm{~d}$ \\
\hline Imazamox + s-metolachlor ${ }^{3}$ & $78 \mathrm{~cd}$ & $98 \mathrm{ab}$ & $93 \mathrm{abcd}$ & $87 \mathrm{abc}$ & $68 \mathrm{bc}$ \\
\hline Bentazon & $0 \mathrm{~g}$ & $0 \mathrm{c}$ & $0 \mathrm{f}$ & $0 \mathrm{f}$ & $0 \mathrm{~d}$ \\
\hline Imazamox & $74 \mathrm{de}$ & $94 \mathrm{ab}$ & $82 \mathrm{~d}$ & $76 \mathrm{~cd}$ & $50 \mathrm{c}$ \\
\hline Bentazon + imazamox & $59 \mathrm{e}$ & $95 \mathrm{ab}$ & $85 \mathrm{bcd}$ & $85 \mathrm{~cd}$ & $68 \mathrm{bc}$ \\
\hline Fluazifop-p-butil + fomesafen & $94 \mathrm{ab}$ & $98 \mathrm{a}$ & $100 \mathrm{a}$ & $95 \mathrm{ab}$ & $89 \mathrm{ab}$ \\
\hline Flumioxazin ${ }^{2}+$ fluazifop-p-butil & $87 \mathrm{abcd}$ & $99 \mathrm{a}$ & $98 \mathrm{abc}$ & $95 \mathrm{ab}$ & $90 \mathrm{ab}$ \\
\hline $\mathrm{CV}(\%)$ & 10,82 & 17,73 & 10,01 & 11,82 & 24,77 \\
\hline
\end{tabular}

${ }^{1}$ Dias após a aplicação dos tratamentos; ${ }^{2}$ Médias seguidas de mesmas letras minúsculas na coluna não diferem entre si pelo teste de Tukey a p $<0,05 ;{ }^{3}$ Aplicação do herbicida em pré-emergência da cultura.

Tabela 4. Controle (\%) de papuã infestante da cultivar de feijão do tipo preto, BRS Campeiro em função da aplicação de herbicidas em pré e pós-emergência.

\begin{tabular}{|c|c|c|c|c|c|}
\hline \multirow{2}{*}{ Tratamentos } & \multicolumn{4}{|c|}{ Controle de papuã (\%) } & \multirow{2}{*}{$\begin{array}{c}\text { Pré } \\
\text { colheita }\end{array}$} \\
\hline & 07 DAT ${ }^{1}$ & 14 DAT & 21 DAT & 28 DAT & \\
\hline Testemunha infestada & $0 \mathrm{~h}^{2}$ & $0 \mathrm{~d}$ & $0 \mathrm{e}$ & $0 \mathrm{~d}$ & $0 \mathrm{~d}$ \\
\hline Testemunha capinada & $100 \mathrm{a}$ & $100 \mathrm{a}$ & $100 \mathrm{a}$ & $100 \mathrm{a}$ & $100 \mathrm{a}$ \\
\hline Fomesafen + s-metolachlor ${ }^{3}$ & 76 de & $69 \mathrm{~b}$ & $61 \mathrm{c}$ & $54 \mathrm{c}$ & $0 \mathrm{~d}$ \\
\hline Oxifluorfen $^{3}$ & $0 \mathrm{~h}$ & $0 \mathrm{~d}$ & $0 \mathrm{e}$ & $0 \mathrm{~d}$ & $0 \mathrm{~d}$ \\
\hline S-metolachlor ${ }^{3}$ & $62 \mathrm{f}$ & $33 \mathrm{c}$ & $33 \mathrm{~d}$ & $43 \mathrm{c}$ & $0 \mathrm{~d}$ \\
\hline Clomazone $^{3}$ & $91 \mathrm{abc}$ & $91 \mathrm{a}$ & $88 \mathrm{~b}$ & $83 \mathrm{~b}$ & $0 \mathrm{~d}$ \\
\hline Pendimenthalin ${ }^{3}$ & $0 \mathrm{~h}$ & $0 \mathrm{~d}$ & $0 \mathrm{e}$ & $0 \mathrm{~d}$ & $0 \mathrm{~d}$ \\
\hline Flumioxazin ${ }^{3}$ & $35 \mathrm{~g}$ & $0 \mathrm{~d}$ & $0 \mathrm{e}$ & $0 \mathrm{~d}$ & $0 \mathrm{~d}$ \\
\hline Fluazifop-p-butil & $92 \mathrm{ab}$ & $98 \mathrm{a}$ & $99 \mathrm{ab}$ & $93 \mathrm{ab}$ & $95 \mathrm{ab}$ \\
\hline Fomesafen & $60 \mathrm{f}$ & $28 \mathrm{c}$ & $31 \mathrm{~d}$ & $0 \mathrm{~d}$ & $0 \mathrm{~d}$ \\
\hline Imazamox + s-metolachlor ${ }^{3}$ & $81 \mathrm{bcd}$ & $96 \mathrm{a}$ & $91 \mathrm{ab}$ & $83 \mathrm{~d}$ & $79 \mathrm{~b}$ \\
\hline Bentazon & $0 \mathrm{~h}$ & $0 \mathrm{~d}$ & $0 \mathrm{e}$ & $0 \mathrm{~d}$ & $0 \mathrm{~d}$ \\
\hline Imazamox & 79 cde & $95 \mathrm{a}$ & $97 \mathrm{ab}$ & $84 \mathrm{~b}$ & $43 \mathrm{c}$ \\
\hline Bentazon + imazamox & 69 ef & $95 \mathrm{a}$ & $92 \mathrm{ab}$ & $88 \mathrm{~b}$ & $77 \mathrm{~b}$ \\
\hline Fluazifop-p-butil + fomesafen & $92 \mathrm{ab}$ & 97 a & $99 \mathrm{ab}$ & $90 \mathrm{ab}$ & $76 \mathrm{~b}$ \\
\hline Flumioxazin 2 + fluazifop-p-butil & $93 \mathrm{a}$ & $99 \mathrm{a}$ & $99 \mathrm{ab}$ & $93 \mathrm{ab}$ & $87 \mathrm{ab}$ \\
\hline $\mathrm{CV}(\%)$ & 8,04 & 6,92 & 8,24 & 9,18 & 23,81 \\
\hline
\end{tabular}

${ }^{1}$ Dias após a aplicação dos tratamentos; ${ }^{2}$ Médias seguidas de mesmas letras minúsculas na coluna não diferem entre si pelo teste de Tukey a $\mathrm{p}<0,05 ;{ }^{3}$ Aplicação do herbicida em pré-emergência da cultura. 
solo - teor e tipo de argila e de matéria orgânica, CTC, $\mathrm{pH}$, dentre outros - além da solubilidade em água das moléculas dos herbicidas (Prata et al., 2003). Procópio et al. (2001) relatam que em solos com baixo teor de matéria orgânica e CTC efetiva, por exemplo, ocorreu aumento de efeitos fitotóxicos do s-metolachlor às culturas e também a probabilidade de contaminação de águas subterrâneas (Procópio et al., 2001).

Alguns trabalhos que avaliaram os herbicidas, s-metolachlor, imazethapyr, imazamox, bentazon, fluazifop-p-buthyl, fomesafen demonstraram em outras cultivares de feijão que apresentam potencial para o manejo de plantas daninhas nessa cultura, tanto em aplicações isoladas quanto em misturas (Soltani et al., 2005; Sikkema et al., 2008; Silva et al., 2014), ou seja, controlaram de modo eficaz as plantas daninhas e foram seletivos ao feijão.
Observou-se que o oxifluorfen foi o mais prejudicial ao feijão ao se analisar o número de plantas (NP) por metro, pois resultou em decréscimo de aproximadamente $34 \%$ em comparação a testemunha capinada (Tabela 5). O oxifluorfen não diferiu significativamente dos herbicidas fomesafen + s-metolachlor, s-metolachlor, pendimenthalin, fomesafen e bentazon. Com exceção do fomesafen, todos os demais são recomendados em aplicações de pré-emergência das plantas daninhas e da cultura (Rodrigues e Almeida, 2011).

O maior número de vagens por plantas (NVP) foi observado na testemunha capinada, não diferindo da aplicação dos herbicidas clomazone, fluazifopp-buthyl, imazamox + s-metolachlor, imazamox, bentazon + imazamox, fluazifop-p-buthyl + fomesafen e flumioxazin + fluazifop-p-buthyl (Tabela 5). Esses resultados são decorrentes

Tabela 5. Componentes de rendimento de grãos da cultivar de feijão do tipo preto, BRS Campeiro, número de plantas por metro, número de vagens por plantas, número de grãos por vagens, massa de mil grãos e produtividade de grãos, em função da aplicação de herbicidas em pré e pós-emergência.

\begin{tabular}{|c|c|c|c|c|c|}
\hline \multirow[b]{2}{*}{ Tratamentos } & \multicolumn{5}{|c|}{ Componentes de rendimento (\%) } \\
\hline & $\begin{array}{l}\text { Número de } \\
\text { plantas } \\
\text { (m) }\end{array}$ & $\begin{array}{c}\text { Número } \\
\text { de vagens } \\
\text { planta }^{-1}\end{array}$ & $\begin{array}{l}\text { Número de } \\
\text { grãos por } \\
\text { vagem }^{-1}\end{array}$ & $\begin{array}{l}\text { Massa de } \\
1000 \text { grãos } \\
\text { (g) }\end{array}$ & $\begin{array}{l}\text { Produtividade } \\
\text { de grãos } \\
\left(\mathrm{kg} \mathrm{ha}^{-1}\right)\end{array}$ \\
\hline Testestemunha. infestada & $8,1 \mathrm{abc}^{1}$ & $2,8 \mathrm{bc}$ & $4,3 \mathrm{c}$ & 152 abcd & $337 \mathrm{f}$ \\
\hline Testemunha capinada & $9,3 \mathrm{a}$ & 7,9 a & $5,2 \mathrm{abc}$ & $173 \mathrm{a}$ & $1436 \mathrm{a}$ \\
\hline Fomesafen + s-metolachlor ${ }^{2}$ & $7,8 \mathrm{abc}$ & $4,3 \mathrm{~b}$ & $5,0 \mathrm{abc}$ & $151 \mathrm{abcd}$ & 669 de \\
\hline Oxifluorfen ${ }^{2}$ & $6,1 \mathrm{c}$ & $2,5 \mathrm{c}$ & $4,4 \mathrm{bc}$ & $143 \mathrm{bcd}$ & $302 \mathrm{f}$ \\
\hline S-metolachlor ${ }^{2}$ & $8,3 \mathrm{abc}$ & $3,3 \mathrm{bc}$ & $4,8 \mathrm{abc}$ & $137 \mathrm{~d}$ & $354 \mathrm{f}$ \\
\hline Clomazone $^{2}$ & $9,0 \mathrm{ab}$ & $6,5 \mathrm{a}$ & $5,3 \mathrm{ab}$ & $162 a b c$ & $985 \mathrm{bc}$ \\
\hline Pendimenthalin ${ }^{2}$ & $6,8 \mathrm{bc}$ & $2,2 \mathrm{c}$ & $4,7 \mathrm{abc}$ & $142 \mathrm{bcd}$ & $204 \mathrm{f}$ \\
\hline Flumioxazin ${ }^{2}$ & $8,4 \mathrm{ab}$ & $3,2 \mathrm{bc}$ & $4,8 \mathrm{abc}$ & $144 \mathrm{bcd}$ & $455 \mathrm{ef}$ \\
\hline Fluazifop-p-butil & $9,5 \mathrm{a}$ & $6,3 \mathrm{a}$ & $5,0 \mathrm{abc}$ & $165 \mathrm{ab}$ & $1126 \mathrm{~b}$ \\
\hline Fomesafen & $8,1 \mathrm{abc}$ & $2,5 \mathrm{c}$ & $4,8 \mathrm{abc}$ & $145 \mathrm{bcd}$ & $382 \mathrm{f}$ \\
\hline Imazamox + s-metolachlor ${ }^{2}$ & $9,9 \mathrm{a}$ & $6,2 \mathrm{a}$ & $4,6 \mathrm{abc}$ & $172 \mathrm{a}$ & $1055 \mathrm{bc}$ \\
\hline Bentazon & $8,1 \mathrm{abc}$ & $2,5 \mathrm{c}$ & $4,9 \mathrm{abc}$ & $139 \mathrm{~cd}$ & $269 \mathrm{f}$ \\
\hline Imazamox & $9,2 \mathrm{ab}$ & $6,3 \mathrm{a}$ & $4,3 \mathrm{c}$ & $158 \mathrm{abcd}$ & $785 \mathrm{~cd}$ \\
\hline Bentazon + imazamox & $9,9 \mathrm{a}$ & $6,5 \mathrm{a}$ & $5,4 \mathrm{a}$ & $173 \mathrm{a}$ & $1444 \mathrm{a}$ \\
\hline Fluazifop-p-butil + fomesafen & $9,8 \mathrm{a}$ & $7,1 \mathrm{a}$ & $5,1 \mathrm{abc}$ & $162 a b c$ & $1080 \mathrm{~b}$ \\
\hline Flumioxazin $^{2}+$ fluazifop-p-butil & $9,0 \mathrm{ab}$ & $7,2 \mathrm{a}$ & $4,7 \mathrm{abc}$ & $169 \mathrm{a}$ & $1159 \mathrm{~b}$ \\
\hline $\mathrm{CV}(\%)$ & 10,64 & 14,89 & 7,99 & 5,92 & 14,31 \\
\hline
\end{tabular}

${ }^{1}$ Médias seguidas de mesmas letras minúsculas na coluna não diferem entre si pelo teste de Tukey a p $<0,05 ;{ }^{2}$ Aplicação do herbicida em pré-emergência da cultura. 
da baixa fitotoxicidade e controle satisfatório das plantas daninhas (Tabela 2 a 4) que esses herbicidas ocasionaram, demonstrando que a falha na aplicação de algum método de controle de plantas daninhas pode reduzir significativamente esse componente de rendimento do feijão do tipo preto. Ao utilizarem o bentazon + imazamox Linhares et al. (2014), chegaram a conclusão que essa mistura não altera as características de crescimento das plantas de feijoeiro, apresentando seletividade e podendo ser usada em feijão para o controle de plantas daninhas.

O número de grãos por vagens (NGV) foi pouco influenciado pelos herbicidas utilizados no presente experimento, sendo inferior somente quando os herbicidas ocasionaram elevadas fitotoxicidade a cultura (oxifluorfen) ou baixo/nenhum controle de papuã e da milhã (imazamox e a testemunha infestada). Lamego et al. (2011) ao estudarem o efeito de s-metolachlor e alachlor em diferentes doses, observaram que o NGV não apresentou diferença significativa, nem mesmo em relação as testemunhas infestada e capinada.

A aplicação do herbicida s-metolachlor foi o que influenciou negativamente na massa de mil grãos (MMG), com redução de $21 \%$ em relação a testemunha capinada. Destaca-se ainda que os demais herbicidas aplicados em pré-emergência, com exceção do clomazone, também apresentaram diminuição desta variável em comparação com a testemunha capinada. Dentre os herbicidas aplicados em pós-emergência, destaca-se o bentazon e o fomesafen como os que também apresentaram o mesmo efeito negativo, como menor MMG ao se comparar com os demais (Tabela 5).

Os resultados demonstram que a maior produtividade de grãos (PROD) foi obtida ao se aplicar a mistura comercial de bentazon + imazamox, a qual se igualou a testemunha capinada (Tabela 5). Esse resultado ocorre pela combinação de fatores como, herbicida com baixa fitotoxicidade ao feijão do tipo preto cultivar BRS Campeiro, bom controle de papuã e de milhã, e reduzido efeito sobre os componentes do rendimento de grãos. Ressalta-se ainda que se torna interessante a combinação dessas duas moléculas herbicidas, por pertencerem a dois mecanismos de ação distintos (inibidor de FS II - bentazon e de ALS - imazamox), o que pode dificultar a seleção de plantas daninhas resistentes ou favorece o manejo de plantas daninhas infestantes do feijão do tipo preto. Lamego et al. (2011) relataram que herbicidas causadores das maiores fitotoxicidades em plantas de feijão ocasionaram como consequência a menor produtividade de grãos.

Os resultados demonstram que a aplicação de fluazifop-p-buthyl de modo isolado ou associado com o fomesafen e o flumioxazin, apresentou resultado satisfatórios para a PROD de grãos (Tabela 5). Contudo não foram eficientes a ponto de manter a cultura livre da competição de papuã e de milhã do início até o final do ciclo, o que ocasionou numa redução média de $22 \%$ na PROD ao se comparar com a testemunha capinada.

A aplicação de herbicidas somente em pré-emergência não foi suficiente para que a cultura viesse a findar o ciclo sem a competição com plantas daninhas, muito provavelmente pelo banco de sementes do solo ser extremamente alto, principalmente de papuã. Porém o herbicida clomazone foi dentre os pré-emergentes o que alcançou a melhor PROD de grãos (Tabela 5), necessitando assim de mais trabalhos, em diferentes locais, para aproveitar seu possível potencial de uso para o manejo de plantas daninhas infestantes do feijão do tipo preto.

As associações de herbicidas em pré e pós-emergência contribuem para minimizar os problemas de resistência das plantas daninhas, pois, introduz na área, na maioria das vezes, mais do que um mecanismo de ação o que pode reduzir casos de espécies resistentes, além de aumentar o espectro de controle de plantas infestantes da lavoura.

\section{Conclusões}

O herbicida oxifluorfen foi o mais fitotóxico a cultivar de feijão do tipo preto, BRS Campeiro e os menos foram o fluazifop-p-buthyl, o fomesafen e o bentazon. 
A combinação dos herbicidas fluazifop-p-buthyl + fomesafen e de flumioxazin + fluazifop-p-buthyl contribui para o manejo das plantas daninhas infestantes do feijão, em especial o papuã e a milhã.

A mistura comercial de bentazon + imazamox foi a que apresentou o menor efeito negativo sobre os componentes de rendimento de grãos do feijão.

O clomazone demonstra ter potencial para uso no controle de plantas infestantes do feijão, necessitando assim de mais estudos para comprovação.

\section{Agradecimentos}

Ao CNPq, à FAPERGS e ao FINEP pelo auxílio financeiro à pesquisa e pelas concessões de bolsas.

\section{Referências}

Armel, G.R.; Hall, G.J.; Wilson, H.P.; Culler, N. Mesotrione plus atrazine mixtures for control of canada thistle (Cirsium arvense). Weed Science, v.53, n.2, p.202-211, 2005.

Borchartt, L.; Jakelaitis, A.; Valadão, F.C.A.; Venturoso, C.L.A.; Santos, C.L. Períodos de interferência de plantas daninhas na cultura do feijoeiro-comum (Phaseolus vulgaris L.). Revista Ciência Agronômica, v.42, n.3, p.725-734, 2011.

Bressanin, F.N.; Giancotti, P.R.F.; Neto, N.J.; Amaral, C.L.; Alves, L.C.A. Eficácia de herbicidas aplicados isolados em pré e pós-emergência no controle de mucuna-preta. Agrária, v.10, n.3, p.426-431, 2015.

Brunharo, C.A.C.G.; Christoffoleti, P.J.; Nicolai, M. Aspectos do mecanismo de ação do amônio glufosinato: culturas resistentes e resistência de plantas daninhas. Revista Brasileira de Herbicidas, v.13, n.2, p.163-177, 2014.

Bunting, J.A.; Sprague, C.; Riechers, D.E. Corn tolerance as affected by the timing of foramsulfuron applications. Weed Technology, v.18, n.3, p.757$762,2004$.
Companhia Nacional de Abastecimento CONAB. Acompanhamento da safra brasileira de grãos. v. 4 - Safra 2016/17, n 5 - Quinto levantamento, fevereiro 2017. Disponível em: $<$ http://www.conab.gov.br/OlalaCMS/uploads/ arquivos/17_02_16_11_51_51_boletim_graos fevereiro_2017.pdf>. Acesso em: 20 fev. 2017.

Empresa Brasileira de Pesquisa Agropecuária EMBRAPA. Sistema brasileiro de classificação de solos. Brasília, DF: Embrapa Solos, 2013. 353p.

Fontana, L.C.; Agostinetto, D.; Dal Magro, T.; Ulguim, A.R.; Canto-Dorow, T.S. Levantamento de espécies de Digitaria ("milhã") em áreas de cultivo agrícola no Rio Grande do Sul (Brasil). Revista Brasileira de Biociências, v.14, n.1, p.1-8, 2016.

Fontes, J.R.A.; Oliveira, I.J.; Gonçalves, J.R.P. Seletividade e eficácia de herbicidas para cultura do feijão-caupi. Revista Brasileira de Herbicidas, v.12, n.1, p.47-55, 2013.

Kalsing, A.; Vidal, R.A. Redução da infestação de papuã (Urochloa plantaginea) no feijoeiro comum através do uso de herbicidas residuais. Planta Daninha, v.30, n.3, p.575-580, 2012.

Lamego, F.P.; Basso, C.J.; Vidal, R.A.; Trezzi, M.M.; Santi, A.L.; Ruchel, Q.; et al. Seletividade dos herbicidas s-metolachlor e alachlor para o feijão-carioca. Planta Daninha, v.29, n.4, p.877883, 2011.

Linhares, C.M.S.; Freitas, F.C.L.; Silva, K.S.; Lima, M.F.P.; Dombroski, J.L.D. Crescimento do feijão-caupi sob efeito dos herbicidas fomesafen e bentazon+imazamox. Revista Caatinga, v.27, n.1, p.41-49, 2014.

Mancuso, M.A.C.; Aires, B.C.; Negrisoli, E.; Corrêa, M.R.; Soratto, R.P. Seletividade e eficiência de herbicidas no controle de plantas daninhas na cultura do feijão-caupi. Revista Ceres, v.63, n.1, p.25-32, 2016.

Nina, N.C.S. Controle de plantas daninhas com herbicidas e efeitos da seletividade destes sobre 
o crescimento e produtividade de feijão-caupi (Vigna unguiculata (L.) Walp.). 2011. $144 \mathrm{f}$. Tese (Doutorado em Agronomia Tropical) - Universidade Federal do Amazonas, Manaus, 2011.

Oliveira, A.R.; Freitas, S.P.; Vieira, H.D. Controle de Commelina benghalensis, C. erecta, Tripogandra diuretica na cultura do café. Planta Daninha, v.27, n.4, p.823-830, 2009.

Oliveira, M.B.; Alves, P.F.S.; Teixeira, M.F.F.; Silva, H.D.; Sá, R.A.; Campos, R.G.C.; et al. Fitotoxicidade de herbicidas aplicados em diferentes épocas em pós-emergência do feijãocaupi. Revista Unimontes Científica, v.15, n.1, p.62-70, 2013.

Prata, F.; Cardinali, V.C.B.; Lavorenti, A.; Tornisielo, V.L.; Reginato, J.B. Glyphosate sorption and desorption in soils with different phosphorous levels. Scientia Agrícola, v.60, n.1, p.175-180, 2003.

Procópio, S.D.; Silva, A.A.; Santos, J.B.; Ferreira, L.R.; Miranda, G.V.; Siqueira, J.G. Efeito da irrigação inicial na profundidade de lixiviação do herbicida s-metolachlor em diferentes tipos de solo. Planta Daninha, v.19, n.3, p.409-417, 2001.

Rodrigues, B.N.; Almeida, F.R. Guia de herbicidas. Londrina: Edição dos Autores, 2011. 697p.

Rede oficial de laboratórios de análise de solo e de tecido vegetal - ROLAS. Manual de adubação e calagem para os estados do Rio Grande do Sul e Santa Catarina. 10.ed. Porto Alegre: Sociedade Brasileira de Ciência do Solo, 2004. 400p.

Santos, L.O.; Pinto, J.J.O.; Piveta, L.B.; Noldin, J.A.; Galon, L.; Concenço, G. Carryover effect of imidazolinone herbicides for crops following rice. American Journal of Plant Sciences, v.5, n. 8, p.1049-1058, 2014.

Sikkema, P.H.; Robinson, D.E.; Nurse, R.E.; Soltani, N. Pre-emergence herbicides for potential use in pinto and small red mexican bean (Phaseolus vulgaris) production. Crop Protection, v.27, n.1, p.124-129, 2008.

Silva, K.S.; Freitas, F.C.L.; Silveira, L.M.; Linhares, C.S.; Carvalho, D.R.; Lima, M.F.P. Eficiência de herbicidas para a cultura do feijão-caupi. Planta Daninha, v.32, n.1, p.197-205, 2014.

Silva, V.P.; Ferreira, L.R.; D'antonino, L.; Carneiro, J.E.; Silva, G.R.; Fontes, D.R. Eficiência e residual no solo de herbicidas na cultura do feijão. Planta Daninha, v.31, n.4, p.961-970, 2013.

Sociedade Brasileira da Ciência das Plantas Daninhas - SBCPD. Procedimentos para instalação, avaliação e análise de experimentos com herbicidas. Londrina: SBCPD, 1995. 42p.

Soltani, N.; Bowley, S.; Sikkema, P.H. Responses of black and cranberry beans (Phaseolus vulgaris) to post-emergence herbicides. Crop Protection, v.24, n.1, p.15-21, 2005.

Vargas, L.; Roman, E.S. Seletividade e eficiência de herbicidas em cereais de inverno. Revista Brasileira de Herbicidas, v.4, n.3, p.1-10, 2005.

Vidal, R.A.; Kalsing, A.; Gherekhloo, J. Interferência e nível de dano econômico de Brachiaria plantaginea e Ipomoea nil na cultura do feijão comum. Ciência Rural, v.40, n.8, p.1675-1681, 2010.

Zargar, M.; Pakina, E.N.; Romanova, E.V. Herbicide doses and application times in weed suppression on different red bean varieties. Procedia, v.8, n.1, p.75-81, 2014. 